\title{
Mechanism of liquid crystal alignment on submicron patterned surfaces
}

\author{
A. Rastegar ${ }^{a)}$ \\ Research Institute for Materials and Nijmegen SON Research Institute for Molecular Structure, Design, \\ and Synthesis, University of Nijmegen, Toernooiveld 1, 6525ED Nijmegen, The Netherlands \\ M. Škarabot \\ Research Institute for Materials, University of Nijmegen, Toernooiveld 1, 6525ED Nijmegen, \\ The Netherlands and University of Ljubljana, Jadranska 19, Ljubljana, Slovenia \\ B. Blij and Th. Rasing \\ Research Institute for Materials, University of Nijmegen, Toernooiveld 1, 6525ED Nijmegen, \\ The Netherlands
}

(Received 27 July 2000; accepted for publication 31 October 2000)

\begin{abstract}
The alignment mechanism of liquid crystals on polymeric surfaces that were patterned using an atomic force microscope (AFM) tip was studied by polarizing optical microscopy. Depending on the thickness of the polymer, polymer chain alignment or grooves appear to be responsible for the liquid crystal alignment. In thick polymer films (above $100 \mathrm{~nm}$ ) the polymer chains will align in the direction of the scan due to the large lateral force exerted by the tip. In thin polymer films $(5-20 \mathrm{~nm})$ the polymer chains are strongly fixed to the surface and will not realign by the tip; instead grooves will be formed. The azimuthal surface anchoring the energy of thick polymer films does not depend on the scan line separation and scan force, is of the same order of magnitude as for conventionally rubbed polyimide surfaces, but increases by the number of scans. AFM patterned pixels do not show a pretilt angle. (C) 2001 American Institute of Physics. [DOI: 10.1063/1.1335649]
\end{abstract}

\section{INTRODUCTION}

The atomic force microscope (AFM) not only enables the investigation of nonconducting surfaces on a nanometer scale, but also allows the manipulation of matter at that scale, including polymer surfaces. ${ }^{1}$ Ruetschi et $a l^{2}{ }^{2}$ showed that liquid crystals (LCs) on AFM scanned polymer surfaces will align in the scan direction. By making a LC waveguide, they showed the ability of this technique to manufacture (sub)micron dimension LC devices. The mechanism of the LC alignment was explained by the appearance of grooves on the scanned surface similar to those on conventionally rubbed polyimide (PI) surfaces.

Due to its simplicity and reliability this rubbing of a polymer-coated surface with fine velvet is the most frequently used technique for the alignment of $\mathrm{LCs}^{3{ }^{3-7}} \mathrm{Al}-$ though successfully applied for the past 30 years for the production of liquid crystal displays, the mechanism of the alignment of LC molecules on such a rubbed substrate is not precisely understood. According to the elastic continuum theory of Berreman ${ }^{8}$ the rubbing induces grooves on the polymer surface and the liquid crystal molecules prefer to align parallel to the direction of the grooves. This explanation was also used by Ruetschi et al. ${ }^{2}$ Geary et al. ${ }^{9}$ suggested the alignment of the molecular chains of the polymers by buffing to be responsible for the LC alignment. According to their theory the molecular chains at the surface of the polymer align by the stress that is induced by the rubbing and the LCs will anchor to these aligned chains. Ultimately the bulk LC alignment will follow in an epitaxial manner. The latter

\footnotetext{
a)Electronic mail: rastegar@sci.kun.nl
}

theory was recently supported by Stohr and Samant ${ }^{10}$ in a near edge x-ray absorption spectroscopy experiment.

In this article we suggest that, depending on the thickness and the mechanical properties of the polymer used, the polymer chain alignment, the grooves or a combination of both are responsible for the alignment of liquid crystals on the scanned polymer surface. However, the mechanism of the alignment of polymer chains by AFM tips is also different from the one suggested by Geary et al. ${ }^{9}$ for the conventionally rubbed PI. Instead of inducing strain, with the AFM scanning the lateral forces exerted by the tip appear to displace the polymer backbone along the scan direction.

Although patterning of a polymer surface by an AFM tip may seem very similar to conventional rubbing, one should keep in mind that there are important differences between these two methods. The applied pressure on the polymer surface at the contact point of the AFM tip is much larger than the one applied by the rubbing fiber. An average tip diameter is between 10 and $50 \mathrm{~nm}$. Here we assume the effective tip diameter to be $20 \mathrm{~nm}$, which is much smaller than the effective diameter of fibers $(\sim 1 \mu \mathrm{m})$ used for conventional rubbing. The typical scan force (vertical) that is applied by an AFM tip to pattern the polymer surface is of the order of $\sim 0.1-1 \mu \mathrm{N}$, which is of the same order as the vertical force per fiber $(\sim 10-20 \mu \mathrm{N})$ for conventional rubbing. ${ }^{11}$ Hence the typical tip pressure on the surface is $P_{t}=0.4 \mu \mathrm{N} /$ $0.0004 \mu \mathrm{m}^{2}=10^{3} \mu \mathrm{N} / \mu \mathrm{m}^{2}$ whereas the average fiber pressure on the surface is $P_{f}=15 \mu \mathrm{N} / \mu \mathrm{m}^{2}$ assuming that the whole fiber is in contact with the polymer layer.

Another important difference between conventional rubbing and AFM patterning is the lateral force. In the case of conventional rubbing the lateral force is about a few New- 
ton's and always smaller than the normal force. The dynamic friction coefficient is approximately $\mu=0.54$ for PI. ${ }^{11}$ In AFM scanning the lateral force can be larger than the vertical force. ${ }^{12}$ The lateral force between the tip and the object is not only a function of the vertical force, but depends strongly on the aspect ratio of the tip, the tilt angle of the cantilever, and the friction coefficient between the tip and surface. At moderate friction coefficients the lateral forces may exceed the normal forces by an order of magnitude for tips with a small apex angle. Finally the lateral force also depends on the scan direction. For backward scans, the maximum lateral forces are limited and significantly lower than in the case of forward scanning.

AFM pictures of the conventionally rubbed PI show that the average groove separation is approximately 100-300 $\mathrm{nm} .{ }^{11}$ However in AFM scanning, one can exactly choose the scan line separation from 0 to $36 \mu \mathrm{m}$ in the case of our AFM apparatus. In addition, the scan trajectory appears to be an important parameter.

\section{EXPERIMENT}

For the AFM patterning, polyimide was deposited on indium-tin-oxide coated glass substrates by spin or dip coating, with film thicknesses of $20-200 \mathrm{~nm}$. The substrates were then baked at $100^{\circ} \mathrm{C}$ for $60 \mathrm{~min}$. A Nanoscope III and a Dimension 3000 atomic force microscope ${ }^{13}$ were used to scan the substrates. Standard silicon nitride tips ${ }^{13}$ with an average tip diameter of $20 \mathrm{~nm}$ were used in constant force contact mode to pattern the surface of the PI. Square patterns of different dimensions were prepared with different line separations. The maximum scan size was $132 \times 132 \mu \mathrm{m}^{2}$ for the Dimension 3000 AFM. Scanning parameters such as scan speed, scan force, scan angle, and number of scan lines were varied.

Twisted nematic (TN) cells of the patterned substrates and conventionally rubbed PI counter plates with thicknesses of 2-6.5 $\mu \mathrm{m}$ were prepared. The cells were filled with $5 \mathrm{CB}$ at room temperature or at temperatures above the nematic phase.

The quality of the alignment was determined by optical microscopy. The contrast of the TN pixels was determined by the analysis of images taken by a 10 bit digital camera. The surface anchoring energy was measured by the cell rotation method ${ }^{14}$ under a polarizing microscope.

\section{RESULTS AND DISCUSSION}

Tapping and contact AFM modes were used to study the topography of the polymer surfaces before and after patterning and conventional rubbing. Figure 1 shows the tapping mode AFM pictures of the PI surface after rubbing and contact mode scanning with AFM. Very well defined grooves produced by rubbing [Fig. 1(a)] and by the AFM tip [Fig. 1(b) ] can be seen in very thin PI films $(5-20 \mathrm{~nm})$. However, we did not see any grooves or periodic structures on the patterned surfaces of 200-nm-thick PI films. Instead we observed typical bumpy polymer surfaces. Hence the existence of grooves on AFM patterned polymer surfaces seems to depend on the thickness of the polymer film used.
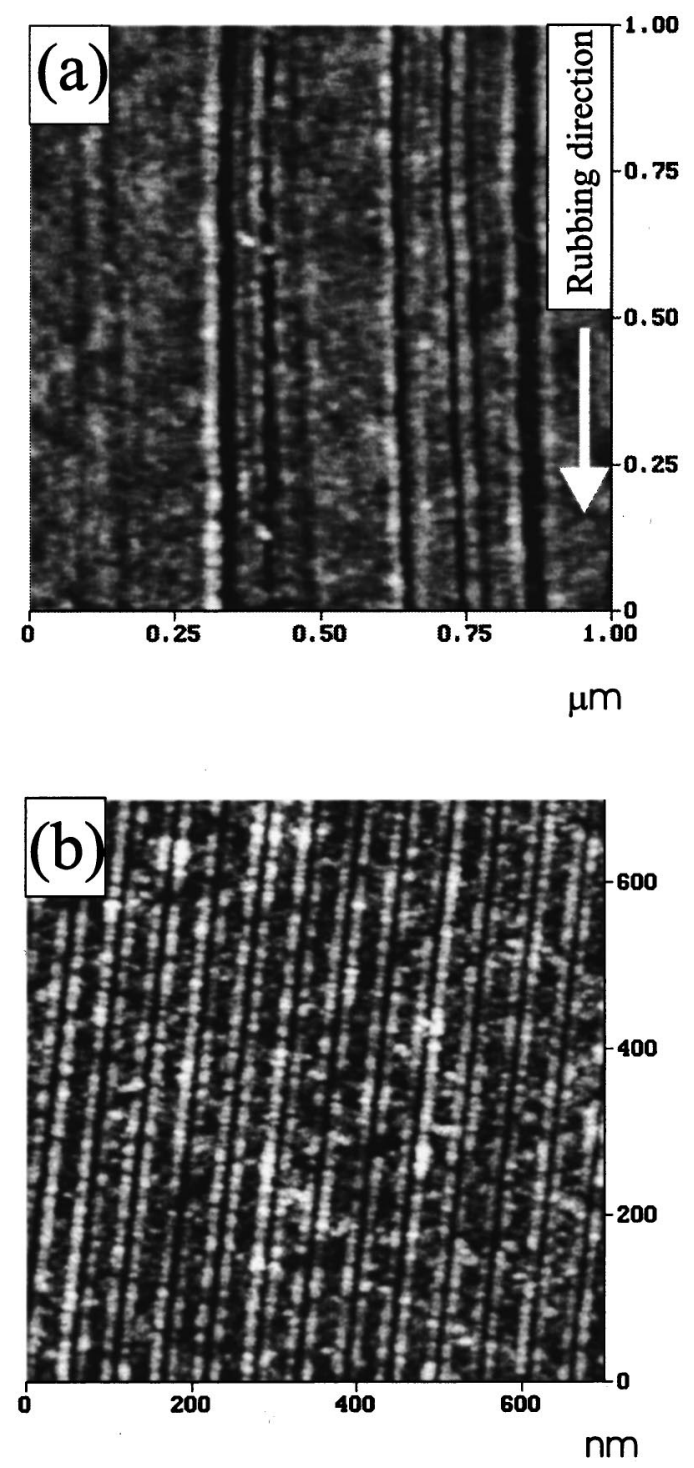

FIG. 1. Tapping mode AFM pictures of the PI surface: (A) after rubbing with velvet, (b) after contact mode scanning with AFM.

PI is a rather soft polymer but its backbone is strong and stiff. On the other hand, as we showed, the tip pressure on the polymer surface could be as large as $10^{9} \mathrm{~N} / \mathrm{m}^{2}$. Therefore the tip can penetrate the polymer surface layer and under the strong lateral force, the polymer chain in contact with the tip will be pulled along in the direction of the displacement of the tip as far as the elastic forces of the polymer will permit. In this way PI chains partly align in the scan direction and consequently LC molecules will align in the direction of the aligned PI chains. However, in the very thin PI films (a few nanometers) surface interactions fix the polymer chains to the surface. The fixed chains cannot be reoriented by the tip, but rather the tip will break the polymer chain and will produce grooves on the surface similar to those observed by Ruetschi et al. ${ }^{2}$

As an example of a polymer with different mechanical properties we studied the alignment properties of poly $(\mathrm{m}-$ ethyl metha acrylate) (PMMA). Conventionally rubbed PMMA aligns nematic liquid crystals. ${ }^{9}$ However, the AFM patterned surfaces would not. PMMA is a side chain polymer 


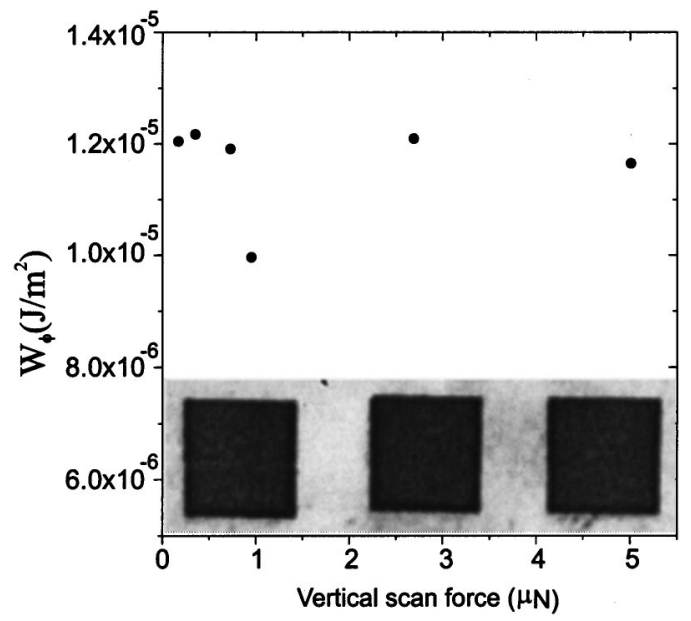

FIG. 2. The azimuthal surface anchoring energy as a function of the scan force in a $3 \mu \mathrm{m}$ cell. The inset shows a micrograph of the patterned (100 $\times 100 \mu \mathrm{m}^{2}$ ) TN pixels under parallel polarizer and analyzer.

that is known to form a helical structure on the surface, which is very brittle. ${ }^{15}$ Hence, during the scanning the tip will not align the polymer chain but break it instead. We also could not see any grooves on the patterned $400 \mathrm{~nm}$ PMMA films by tapping mode AFM. As a result this patterned PMMA surface is not expected to align LCs as was indeed confirmed by our experiments.

Therefore both the polymer film thickness and its mechanical properties are important factors in determining the mechanism of LC alignment. As the alignment of LC on grooved thin films is discussed elsewhere, ${ }^{2}$ we focus our discussion mostly on the mechanism of the alignment of LC in the thick PI polymer films when the polymer chain alignment is thought to be the dominant mechanism.

The azimuthal surface anchoring energy $W_{\phi}$ is a measure for the degree of alignment of liquid crystals on the patterned surfaces. For the thick PI polymer films, by varying the scan force between 0.2 and $0.7 \mu \mathrm{N}$, we found that $W_{\phi}$ is of the same order as for the conventionally rubbed $\operatorname{PI}\left(W_{\phi}=1 \times 10^{-5} \mathrm{~J} / \mathrm{m}^{2}\right)$ and does not depend on the vertical scan force (Fig. 2). This can easily be understood: Increasing the scan force means pushing the tip more into the bulk of the polymer. Therefore not only the surface chains will align but also the chains deeper in the bulk, but these are not effective for the LC alignment. As a result, increasing the scan force will not change the contrast of the TN pixels, as we observed (see the insets of Fig. 2), although it does affect the average depth of the grooves (Fig. 3).

Increasing the number of scans results in the alignment of more polymer chains or a better alignment of the chains that were already partly aligned, and consequently will improve the LC alignment. This can be seen in the increasing azimuthal surface anchoring (Fig. 4) and in the slight increase of the contrast of the TN pixels in the inset of Fig. 4.

Note that increasing the number of scans with an AFM tip is different from increasing the number of rubbings for the conventional alignment. The latter case is similar to reducing the line separation in AFM scanned patterns, which does not affect $W_{\phi}$. In contrast, by increasing the number of scans, the AFM tip will scan the same line a few times
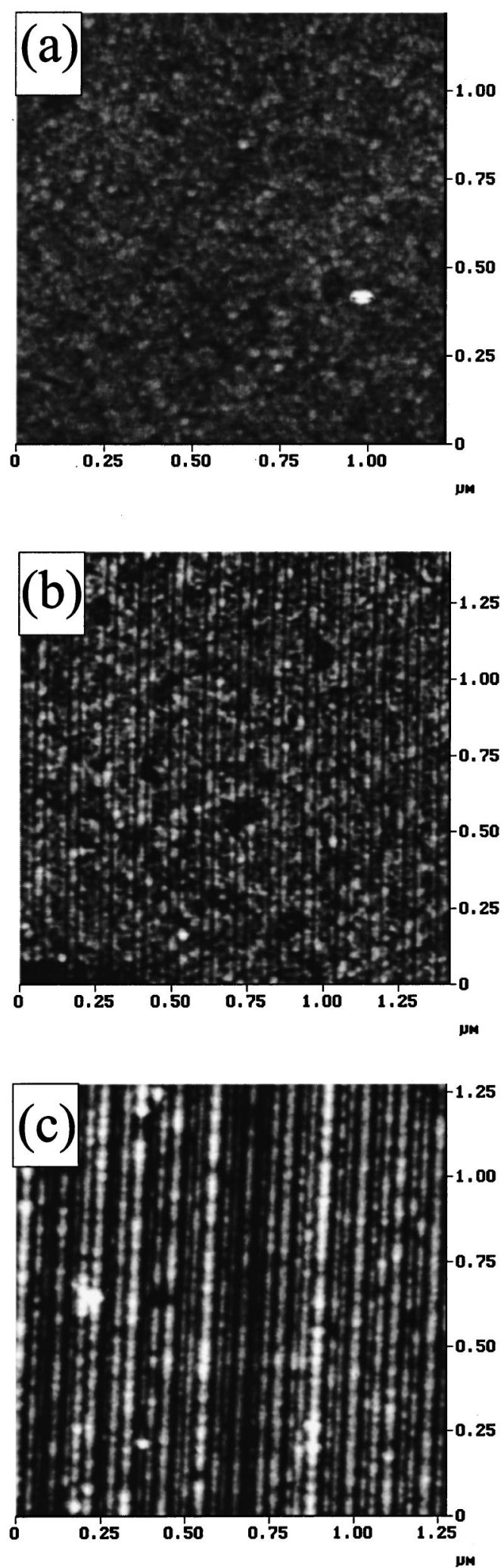

FIG. 3. Tapping mode images of $80 \mathrm{~nm}$ polymer films. (a) Scan force $=0.25 \mu \mathrm{N}$. (b) Scan force $=0.65 \mu \mathrm{N}$. (c) Scan force $=1.5 \mu \mathrm{N}$.

(within a small displacement due to drift) and as a result the polymer chains will align better by each scan.

Finally, when a patterned area is scanned in a different direction, the tip will redirect the polymer chains in the direction of the last scan. Therefore the liquid crystals will 


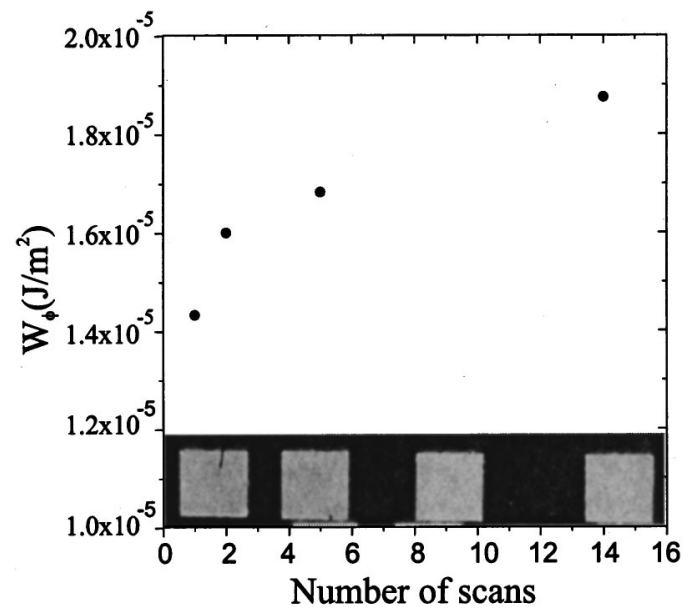

FIG. 4. Azimuthal surface anchoring as a function of the number of scans. The inset shows a micrograph of the patterned $\left(100 \times 100 \mu \mathrm{m}^{2}\right) \mathrm{TN}$ pixels under crossed polarizers

align parallel to the direction of the last scan (see Fig. 5) confirming that the polymer chain alignment is the dominant mechanism of the alignment of LCs on thick polymer films.

The final important problem to address is the existence of a pretilt angle. According to Geary et al. the pretilt angle of a rubbed PI surface is the result of the induced strain that causes the LC molecules to align with a pretilt angle in the direction of the strain. However, in the case of AFM patterning on thick polymer films one does not expect the appearance of a pretilt angle because there is no force to align a chain or a segment of a chain with an angle with respect to the scanning plane.

Figure 6 shows a micrograph of TN pixels under crossed polarizers. The absence of a pretilt causes the formation of multidomains in the whole area of the pixel, as shown in Fig. 6(a). In Fig. 6(b), the scan line separation $d$ is much larger

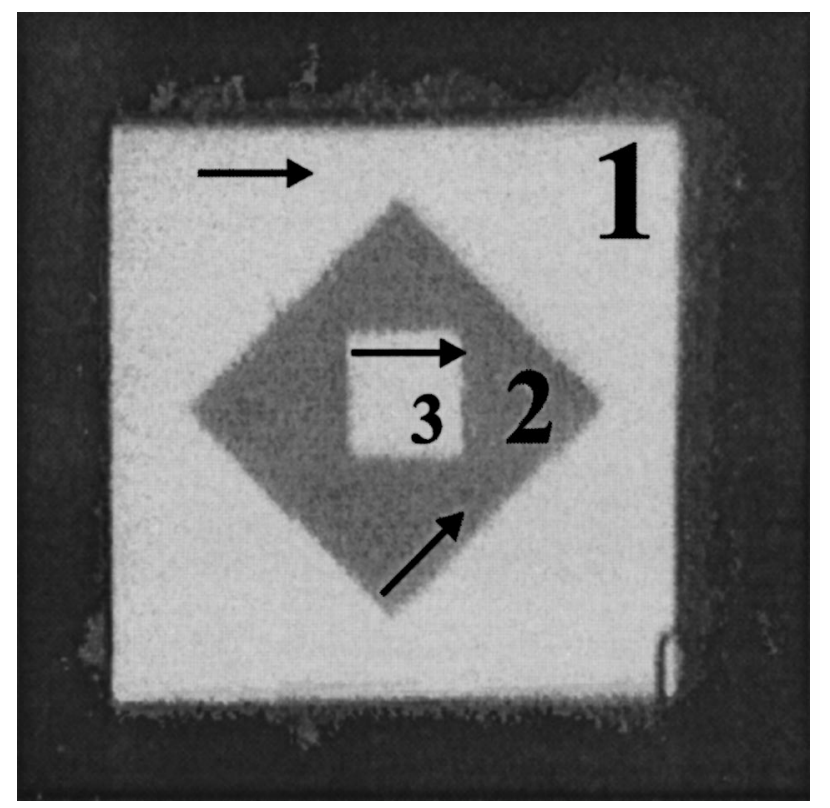

FIG. 5. Micrograph of a patterned TN pixel under crossed polarizers. Arrows indicate the scan direction and the pixel number shows the scan order. The total scanned area is $118 \times 118 \mu \mathrm{m}^{2}$. The LCs follow the direction of the last scan.
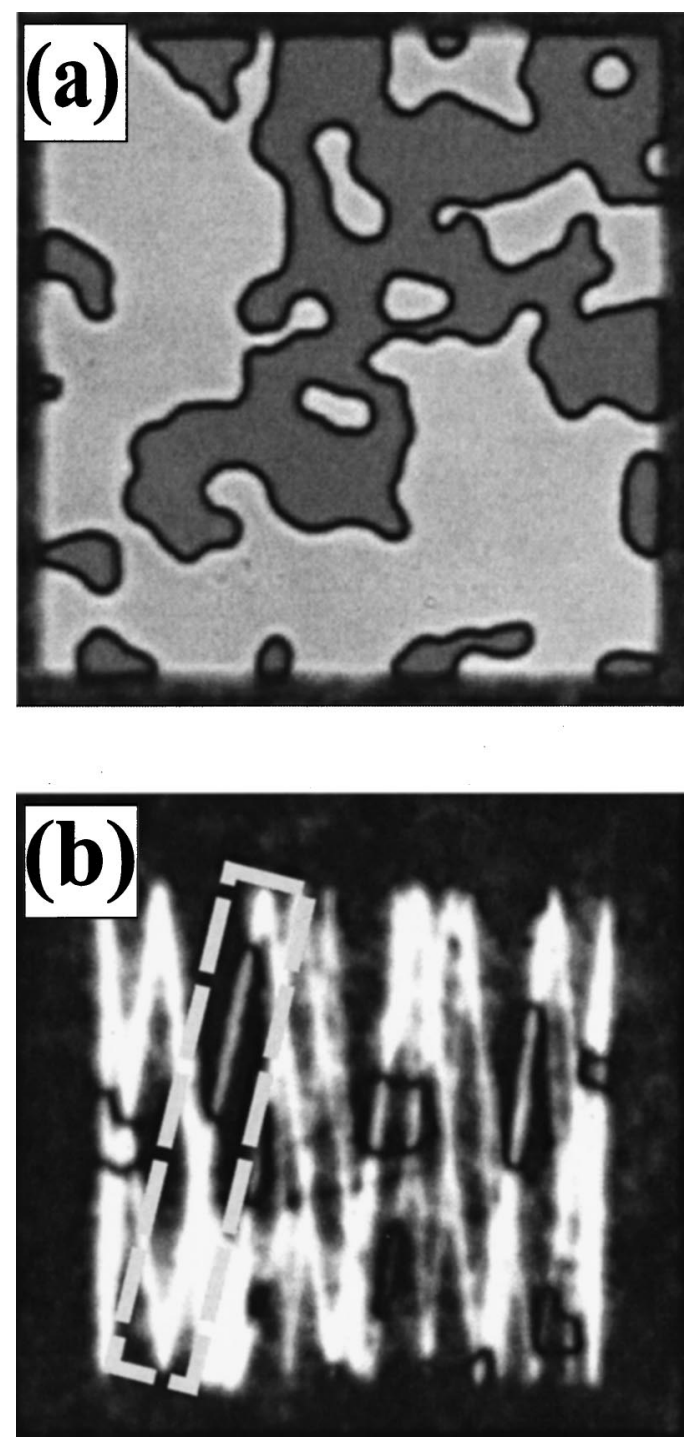

FIG. 6. Micrograph of TN pixels of $100 \mu \mathrm{m} \times 100 \mu \mathrm{m}^{2}$. (a) When the line separation is less than the LC transverse correlation length. Different colors show multiple twisted domains in the TN structure. (B) When the line separation is much larger than the $\mathrm{LC}$ transverse correlation length. The dashed rectangle shows multiple twisted domains in a single line in TN structure.

than the transverse correlation length $\left(\xi_{\perp}\right)$ of the LC. Therefore the LCs will not align homogeneously over the whole patterned area, but they will orient along the direction of the scan line. Consequently, as observed in Fig. 6(b), multidomains are formed even on a single line in the TN structure. This unambiguously indicates that there is no pretilt angle on the patterned surface to remove the twist degeneracy, which is in agreement with the interpretation of the AFM tip aligning the polymer chains.

In conclusion we have shown that AFM patterned polymer surfaces strongly align nematic liquid crystals. On polymer surfaces the anisotropic interaction of LC molecules and polymer chains is responsible for the alignment. Depending on the thickness and mechanical properties of the polymer film, polymer chain alignment and/or grooves are responsible for the LC alignment. However, we suggest a different alignment mechanism for the polymer chains than the one suggested by Geary et al. On the patterned PI surface the 
AFM tip will physically align the polymer chains in the scan direction. The surface anchoring energy increases by increasing the number of scans but remains unchanged by increasing the scan force or reducing the line separation. AFM scanned polymer surfaces do not show any pretilt angle, indicating the absence of tip-induced stress.

\section{ACKNOWLEDGMENTS}

The Solid State Chemistry group (Professor E. Vlieg) of the University of Nijmegen and ing. J. Gerritsen are acknowledged for their assistance with the AFMs. Part of this work was supported by the EU network SILC.

${ }^{1}$ O. M. Leung and M. C. Goh, Science 255, 64 (1992).

${ }^{2}$ M. Ruetschi, P. Grutter, J. Funfschiling, and H.-J. Guntherodt, Science 256, 512 (1994).

${ }^{3}$ M. P. Mahajan and Ch. Rosenblatt, J. Appl. Phys. 83, 7649 (1998).
${ }^{4}$ S. Faetti, Phys. Rev. A 36, 408 (1987).

${ }^{5}$ M. Barmentlo, N. A. J. M. van Aerle, R. W. J. Hollering, and J. P. M. Damen, J. Appl. Phys. 71, 4799 (1992).

${ }^{6}$ K. Sakamoto, R. Arafune, N. Ito, S. Ushioda, Y. Suzuki, and S. Morokawa, J. Appl. Phys. 80, 431 (1996).

${ }^{7}$ J. Cognard, Mol. Cryst. Liq. Cryst. Suppl. Ser. 1, 1 (1982).

${ }^{8}$ D. W. Berreman, Phys. Rev. Lett. 28, 1683 (1972).

${ }^{9}$ J. M. Geary, J. W. Goodby, A. R. Kmetz, and J. S. Patel, J. Appl. Phys. 62, 4100 (1987).

${ }^{10}$ J. Stohr and M. G. Samant, J. Electron Spectrosc. Relat. Phenom. 98-99, 189 (1999).

${ }^{11}$ A. J. Pidduck, G. P. Brayan-Brown, S. D. Haslam, and R. Bannister, Liq. Cryst. 21, 759 (1996).

${ }^{12}$ M. Benoit, T. Holstein, and H. E. Gaub, Eur. Biophys. J. 26, 283 (1997).

${ }^{13}$ Digital Instruments, Veeco Metrology Group, 112 Robin Hill Road, Santa Barbara, CA 93117.

${ }^{14}$ G. P. Bryan-Brown and I. C. Sage, Liq. Cryst. 20, 825 (1996).

${ }^{15}$ H.-M. Wu, Y.-M. Zhu, X.-M. Yang, Q. Luo, Z.-H. Lu, and Y. Wei, Phys. Lett. A 205, 290 (1995). 